\title{
DISTRIBUTION OF THE NUMBER OF HANDOVERS IN A CELLULAR MOBILE COMMUNICATION NETWORK: DELAYED RENEWAL PROCESS APPROACH
}

\author{
Ramón Martín Rodríguez-Dagnino \\ Monterrey Institute of Technology (ITESM)
}

\author{
Hideaki Takagi \\ University of Tsukuba
}

(Received November 14, 2003; Revised September 29, 2004)

\begin{abstract}
Knowing the distribution of the number of handovers during a call session of a given user is particularly important in cellular mobile communication networks in order to make appropriate dimensioning of virtual circuits for wireless cells. In this paper, we study the probability distributions and statistical moments for the number of handovers per call for a variety of combinations of the call holding time (CHT) and cell residence time (CRT) distributions. We assume that the first CRT in the originating cell has different statistics from the CRTs in the subsequent cells. In particular, we consider circular cells. Based on the formulation in terms of delayed renewal processes, we obtain analytical expressions for the probability mass functions and moments of the handover number distribution. We also include some numerical results for the mean number of handovers.
\end{abstract}

Keywords: Telecommunication, cellular network, handover, renewal theory, delayed renewal process

\section{Introduction}

In cellular wireless communication networks, the wireless cells have limited coverage range, which means that mobile users will be crossing several cell boundaries during his call duration. Each boundary crossing may need switching of a communication channel, whether it is a frequency band, a time slot or a code, which is necessary in order to maintain the connectivity with the network as well as the tracking of the location of the user. This cell switching, or handover, tends to cause connectivity disruption and/or extra transmission delay if it is not handled properly. In multimedia service environment, the connectivity and timely transmission are essential. For instance, real-time audio and video information is less tolerant to both overall delay and delay variation than traditional voice calls. Thus, the traffic disruption/delay, as a result of handover failure, can seriously affect the Quality of Service (QoS) of the network.

Wireless multimedia networks are intended to be direct extension of the fixed/wireline broadband ATM networks with uniform end-to-end QoS guarantee. The handover traffic in cellular wireless networks is a complex function of many factors such as the size of wireless cells, user's mobility and call patterns, etc. However it has a direct impact on the signaling traffic, the call admission policy for new users, and also the QoS for the admitted users. Therefore, the study of the handover process is a fundamental issue in the design of multimedia cellular wireless communication networks.

In this paper, we study the statistical characteristics of the number of handovers that a mobile user makes during a call session in a cellular network for a variety of combinations of the call holding time (CHT) and cell residence time (CRT) distributions. In the past, 
Nanda [11] considered the case of exponentially distributed CHT and CRT. The same case has been extensively studied by Lin et al. [9]. However, the CHT cannot be assumed as an exponentially distributed random variable in multimedia services which typically have long tail in distribution. The CRT is not exponentially distributed either. Recently, Fang et al. $[3,4]$ have derived a set of recursive equations for the Erlang distributed CHT. RodríguezDagnino [13] has initiated an innovative method based on the renewal theory for obtaining the distribution and moments of the number of handovers explicitly for a mixture of Erlang (including exponential) distributions for the CHT; this work is elaborated later in $[15$, 17]. However, these studies only consider a homogeneous environment where the same distribution is assumed for the CRTs in all the wireless cells. Recently, Orlik and Rappaport [12] have presented some results for a mixed platform where there are several kinds of mobile users with different speeds such as bicycle, pedestrian, and automobile simultaneously in the network.

In the present paper, we extend the methodology of $[13,15,17]$ to the case where the first CRT and the subsequent CRTs may have different distributions. We derive closed-form expressions for the probability distribution and the moments of the number of handovers per call [14]. In our previous paper [16] we have demonstrated that the CHT is more influential than the CRT in location management applications. Thus we consider the number of handovers when the CHT is generally distributed and the CRT is exponentially distributed. We also extend our methodology to the case in which the third and further subsequent cells have different CRT distributions.

Our approach is based on the renewal theory. Let $N(t)$ be the number of renewals in a fixed time interval $[0, t]$, and the interrenewal times occur according to a sequence of random variables $\left\{X_{1}, X_{2}, \ldots, X_{i}, \ldots\right\}$, where $X_{1}$ is started at time 0 . This represents the sequence of CRTs that a mobile user experiences during a call such that $X_{i}$ is the CRT in the $i$ th cell $(i=1,2, \ldots)$. Now, let $T$ be a random variable representing a CHT; throughout the paper we do not take into account the forced termination of calls due to the blocking of handover process. Let us also assume that $T$ is independent of $\left\{X_{1}, X_{2}, \ldots, X_{i}, \ldots\right\}$. Hence $N(T)$ is a random variable which represents the number of renewals (handovers) in a random interval $[0, T]$ (a CHT). The problem of finding the probability distribution of $N(T)$ has been solved in several specific cases by Cox in his monograph [2, sec. 3.4] under the title "The number of renewals in a random time." Most of the results presented by Cox are based on the ordinary renewal process, i.e., all the random variables $X_{i} ; i=1,2, \ldots$, come from the same distribution [2, p.25]. However, a common situation in cellular networks is that a mobile user begins his call somewhere inside a cell. Thus it is more appropriate to consider the case in which only $X_{i} ; i=2,3, \ldots$, come from the same distribution as a random variable $X_{2}$ while $X_{1}$ may come from a different distribution. Such a case is called the modified or delayed renewal process $[2$, p.28]. This is just the process that we will use as a model of the sequence of CRTs in this paper. As a special case of the delayed renewal process, if $X_{1}$ is a residual life of $X_{2}$, we have the equilibrium renewal process [2, p.28]. This case has been studied by Lin $[8]$ and the present authors $[13,15,17]$. As a generalization of the delayed renewal process, we may assume that each CRT $X_{1}, X_{2}, \ldots$ may have different distribution. See Figure 1 for the diagram of a CHT and CRTs associated with a mobile user in a cellular network.

The remainder of this paper is organized as follows. In Section 2 we present the basic methodology to calculate the probability generating function ( $p g f)$ for $N(T)$ for the CHT with Erlang distribution and for the CRT with any distribution. We consider special cases in which the CHT is exponentially distributed. We derive explicit expressions for the prob- 


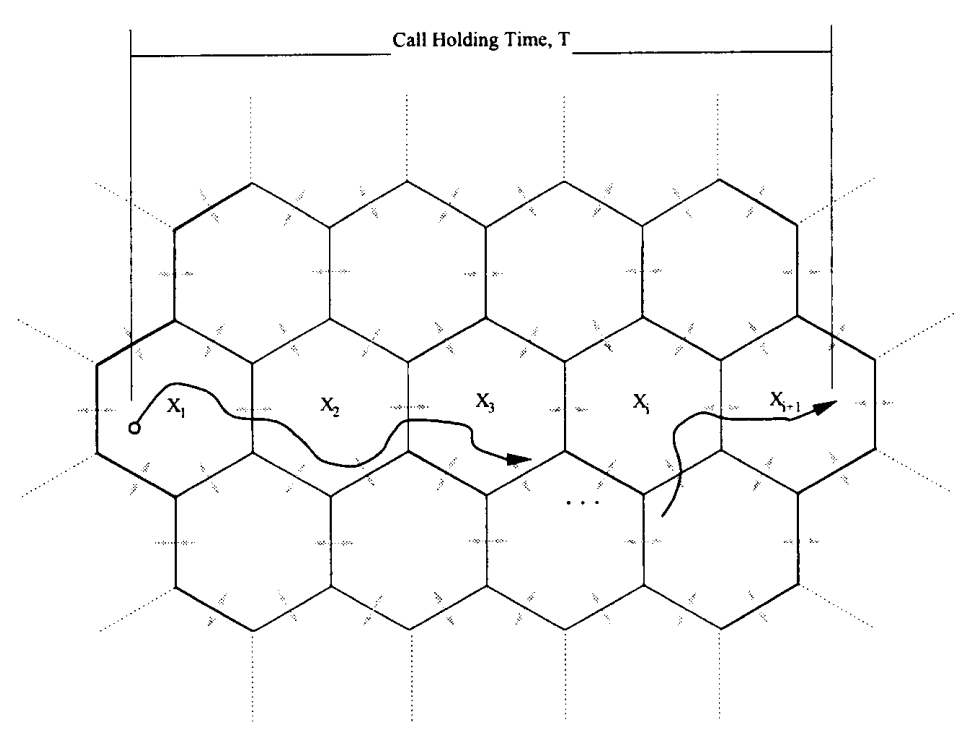

Figure 1: User mobility in a cellular network

ability mass function (pmf) $P[N(T)=j]$ as well as the binomial moments $E\left[\left(\begin{array}{c}N(T) \\ \ell\end{array}\right)\right]$ of $N(T)$ in terms of the Laplace transforms of the probability density function $(p d f)$ for $X_{1}$ and $X_{2}$. In particular, we give simple results for the case of exponentially distributed CRTs. In Section 3, we extend the method of Section 2 to the CHT with a mixture of several exponential distributions, and present closed-form expressions for the $p m f$ and the binomial moments of $N(T)$ when the CRT has exponential, gamma, and generalized gamma distributions. We also analyze the case of circular cells such that the first CRT $X_{1}$ corresponds to the distance from an arbitrary point in a circle to its perimeter in an arbitrary direction and the subsequent CRTs $X_{2}, X_{3}, \ldots$ correspond to the length of a straight line segment cut by a circle. We call this case the circularly distributed CRT. In Section 4 we treat the case in which each CRT $X_{1}, X_{2}, \ldots$ may have different distribution, which we call the generalized delayed renewal process. We study the case in which the CHT is exponentially distributed as well as the case in which the CHT is general and the CRTs are exponentially distributed. We also consider as a special case the delayed renewal process in which the the first CRT $X_{1}$ is exponentially distributed with parameter $\mu_{1}$ and the subsequent CRTs $X_{2}, X_{3}, \ldots$ have the same exponential distribution with parameter $\mu_{2}$, while the CHT has arbitrary distribution. In such a case, the $p m f$ and the binomial moments of $N(T)$ can be expressed in terms of the Laplace transform of the $p d f$ for $T$. In Section 5 some numerical values are shown for $\mathbf{E}[N(T)]$, the mean number of handovers, for a representative set of parameter values. Finally, concluding remarks are made in Section 6.

\section{The pgf Method for Erlang CHT}

Let $G_{N(T)}(z)$ be the $p g f$ for $N(T)$, the number of handovers in a random interval $[0, T]$, where $T$ represents a CHT. It is given by

$$
G_{N(T)}(z)=\int_{t=0}^{\infty} G_{N(T)}(t, z) f_{T}(t) d t
$$

where $f_{T}(t)$ is the $p d f$ of the random variable $T$, and

$$
G_{N(T)}(t, z):=\mathbf{E}\left[z^{N(t)}\right]=\sum_{j=0}^{\infty} P[N(T)=j \mid T=t] z^{j}
$$


is the pgf of $N(t)$, the number of handovers in a fixed interval $[0, t]$. Once $G_{N(T)}(z)$ is obtained, the pmf of $N(T)$ is given by

$$
P[N(T)=j]=\left.\frac{1}{j !} \frac{d^{j}}{d z^{j}} G_{N(T)}(z)\right|_{z=0} ; j=0,1,2, \ldots
$$

The $\ell$ th binomial moment of $N(T)$ is given by

$$
\mathbf{E}\left[\left(\begin{array}{c}
N(T) \\
\ell
\end{array}\right)\right]=\left.\frac{1}{\ell !} \frac{d^{\ell}}{d z^{\ell}} G_{N(T)}(z)\right|_{z=1} ; \quad \ell=0,1,2, \ldots
$$

Let us consider a special case in which the CHT can be fitted by a $k$-stage Erlang $p d f$, say

$$
f_{T}(t)=\frac{\lambda^{k} t^{k-1} e^{-\lambda t}}{(k-1) !} ; \quad t \geq 0
$$

with mean $\mathbf{E}[T]=k / \lambda$. In this case the relation between $G_{N(T)}(z)$ and $G_{N(t)}(t, z)$ has been solved by Cox $[2$, p.43, eq.(4)] as

$$
G_{N(T)}(z)=\left.\frac{\lambda^{k}}{(k-1) !}\left(-\frac{\partial}{\partial s}\right)^{k-1}\left\{G_{N(T)}^{*}(s, z)\right\}\right|_{s=\lambda}
$$

where $G_{N(T)}^{*}(s, z)$ is the Laplace transform of $G_{N(T)}(t, z)$ defined by

$$
G_{N(T)}^{*}(s, z):=\int_{t=0}^{\infty} e^{-s t} G_{N(T)}(t, z) d t
$$

For the delayed renewal process $\left\{X_{1}, X_{2}, \ldots\right\}$ of CRTs, we have [2, p.38, eq.(5)]

$$
G_{N(T)}^{*}(s, z)=\frac{1}{s}+\frac{(z-1) f_{X_{1}}^{*}(s)}{s\left[1-z f_{X_{2}}^{*}(s)\right]}
$$

where $f_{X}^{*}(s)$ is the Laplace transform of the $p d f f_{X}(x)$ for the random variable $X$. Hence we get

$$
\begin{aligned}
G_{N(T)}(z) & =\left.\frac{\lambda^{k}}{(k-1) !}\left(-\frac{\partial}{\partial s}\right)^{k-1}\left\{\frac{1}{s}+\frac{(z-1) f_{X_{1}}^{*}(s)}{s\left[1-z f_{X_{2}}^{*}(s)\right]}\right\}\right|_{s=\lambda} \\
& =1+\left.\frac{\lambda^{k}(z-1)}{(k-1) !}\left(-\frac{\partial}{\partial s}\right)^{k-1} \frac{f_{X_{1}}^{*}(s)}{s\left[1-z f_{X_{2}}^{*}(s)\right]}\right|_{s=\lambda} .
\end{aligned}
$$

From this we can express the pmf of $N(T)$ as

$$
P[N(T)=j]=\left\{\begin{array}{ll}
1-\left.\frac{\lambda^{k}}{(k-1) !}\left(-\frac{\partial}{\partial s}\right)^{k-1} \frac{f_{X_{1}}^{*}(s)}{s}\right|_{s=\lambda} & ; j=0 \\
\left.\frac{\lambda^{k}}{(k-1) !}\left(-\frac{\partial}{\partial s}\right)^{k-1} \frac{f_{X_{1}}^{*}(s)}{s}\left[1-f_{X_{2}}^{*}(s)\right]\left[f_{X_{2}}^{*}(s)\right]^{j-1}\right|_{s=\lambda} & ; j=1,2, \ldots
\end{array} .\right.
$$


We can also express the $\ell$ th binomial moment of $N(T)$ as

$$
\mathbf{E}\left[\left(\begin{array}{c}
N(T) \\
\ell
\end{array}\right)\right]=\left.\frac{\lambda^{k}}{(k-1) !}\left(-\frac{\partial}{\partial s}\right)^{k-1} \frac{f_{X_{1}}^{*}(s)\left[f_{X_{2}}^{*}(s)\right]^{\ell-1}}{s\left[1-f_{X_{2}}^{*}(s)\right]^{\ell}}\right|_{s=\lambda} \quad \ell=1,2, \ldots
$$

We note that if $f_{X_{1}}^{*}(s) \equiv f_{X_{2}}^{*}(s)$, we have an ordinary renewal process for the sequence of CRTs. On the other hand, if

$$
f_{X_{1}}^{*}(s) \equiv \frac{1-f_{X_{2}}^{*}(s)}{\mathbf{E}\left[X_{2}\right] s}
$$

we have the equilibrium renewal process. The latter case has been studied extensively by the authors in [17].

\subsection{Exponentially distributed CHT and general CRT}

Assume that the CHT T is modeled by an exponential $p d f$, say

$$
f_{T}(t)=\lambda e^{-\lambda t} ; \quad t \geq 0
$$

with mean $\mathbf{E}[T]=1 / \lambda$. Then the $p g f$ of $N(T)$ is given by

$$
G_{N(T)}(z)=\left.\lambda\left\{G_{N(T)}^{*}(s, z)\right\}\right|_{s=\lambda}=1+\frac{(z-1) f_{X_{1}}^{*}(\lambda)}{1-z f_{X_{2}}^{*}(\lambda)} .
$$

The $j$ th derivative of this $p g f$ is given by

$$
\frac{d^{j}}{d z^{j}} G_{N(T)}(z)=\frac{j ! f_{X_{1}}^{*}(\lambda)\left[1-f_{X_{2}}^{*}(\lambda)\right]\left[f_{X_{2}}^{*}(\lambda)\right]^{j-1}}{\left[1-z f_{X_{2}}^{*}(\lambda)\right]^{j+1}} ; \quad j=1,2, \ldots
$$

Substituting (2.15) into (2.3), we obtain the pmf of $N(T)$ as

$$
P[N(T)=j]=\left\{\begin{array}{ll}
1-f_{X_{1}}^{*}(\lambda) & ; j=0 \\
f_{X_{1}}^{*}(\lambda)\left[1-f_{X_{2}}^{*}(\lambda)\right]\left[f_{X_{2}}^{*}(\lambda)\right]^{j-1} & ; j=1,2, \ldots
\end{array} .\right.
$$

Substituting (2.15) into (2.4), we obtain the $\ell$ th binomial moment of $N(T)$ as

$$
\mathbf{E}\left[\left(\begin{array}{c}
N(T) \\
\ell
\end{array}\right)\right]=\frac{f_{X_{1}}^{*}(\lambda)\left[f_{X_{2}}^{*}(\lambda)\right]^{\ell-1}}{\left[1-f_{X_{2}}^{*}(\lambda)\right]^{\ell}} \quad \ell=1,2, \ldots
$$

In particular, we have the mean

$$
\mathbf{E}[N(T)]=\frac{f_{X_{1}}^{*}(\lambda)}{1-f_{X_{2}}^{*}(\lambda)}
$$

The variance is given by

$$
\operatorname{Var}[N(T)]=\frac{2 f_{X_{1}}^{*}(\lambda) f_{X_{2}}^{*}(\lambda)}{\left[1-f_{X_{2}}^{*}(\lambda)\right]^{2}}+\mathbf{E}[N(T)]-\mathbf{E}^{2}[N(T)]
$$

We can study several interesting cases by specifying the $p d f$ for both types of CRTs. However, let us defer the most results until Section 3 where we deal with a mixture of exponential distributions for the CHT. In the following subsection, we only consider the case of exponentially distributed CRTs as it reduces to a particularly simple result. 


\subsection{Exponentially distributed CHT and CRTs}

Let us assume that the first CRT $X_{1}$ is exponentially distributed with mean $\mathbf{E}\left[X_{1}\right]=1 / \mu_{1}$, and the subsequent CRTs, each being represented by $X_{2}$, are also exponentially distributed with mean $\mathbf{E}\left[X_{2}\right]=1 / \mu_{2}$. The corresponding Laplace transforms are given by

$$
f_{X_{r}}^{*}(s)=\frac{\mu_{r}}{s+\mu_{r}} ; \quad r=1,2
$$

In this case we have

$$
G_{N(T)}^{*}(s, z)=\frac{s+\mu_{2}+\left(\mu_{1}-\mu_{2}\right) z}{\left(s+\mu_{1}\right)\left(s+\mu_{2}-\mu_{2} z\right)}
$$

and

$$
G_{N(T)}(z)=\frac{1+\rho_{2}+\left(\rho_{1}-\rho_{2}\right) z}{\left(1+\rho_{1}\right)\left(1+\rho_{2}-\rho_{2} z\right)},
$$

where $\rho_{r}=\mathbf{E}[T] / \mathbf{E}\left[X_{r}\right]=\mu_{r} / \lambda$ for $r=1,2$. Hence, the $p m f$ in (2.16) reduces to

$$
P[N(T)=j]=\left\{\begin{array}{ll}
\frac{1}{1+\rho_{1}} & ; j=0 \\
\left(\frac{\rho_{1}}{1+\rho_{1}}\right)\left(\frac{1}{1+\rho_{2}}\right)\left(\frac{\rho_{2}}{1+\rho_{2}}\right)^{j-1} \quad ; j=1,2, \ldots
\end{array} .\right.
$$

The $\ell$ th binomial moment of $N(T)$ is given by

$$
\mathrm{E}\left[\left(\begin{array}{c}
N(T) \\
\ell
\end{array}\right)\right]=\rho_{1}\left(\frac{1+\rho_{2}}{1+\rho_{1}}\right) \rho_{2}{ }^{\ell-1} ; \quad \ell=1,2, \ldots
$$

Therefore, we have

$$
\mathbf{E}[N(T)]=\rho_{1}\left(\frac{1+\rho_{2}}{1+\rho_{1}}\right) \quad ; \quad \operatorname{Var}[N(T)]=\frac{\rho_{1}\left(1+\rho_{2}\right)\left(1+2 \rho_{2}+\rho_{1} \rho_{2}\right)}{\left(1+\rho_{1}\right)^{2}}
$$

\section{Hyperexponentially Distributed CHT and General CRT}

Let us first assume that the CHT $T$ is well modeled by a mixture of $M$ Erlang distributions, say

$$
f_{T}(t)=\sum_{i=1}^{M} p_{i} \frac{\lambda_{i}^{k_{i}} t^{k_{i}-1} e^{-\lambda_{i} t}}{\left(k_{i}-1\right) !} ; \quad t \geq 0
$$

where $\sum_{i=1}^{M} p_{i}=1$, and $k_{i} ; i=1,2, \ldots, M$, is a positive integer. This model may represent a situation such that there are several applications each user can choose from, such as the voice conversation, data transmission for making ticket reservation, and browsing www information on his mobile phone. Application $i$ is chosen with probability $p_{i} ; i=1,2, \ldots, M$. The $p g f$ of $N(T)$ is then given by

$$
G_{N(T)}(z)=\left.\sum_{i=1}^{M} p_{i} \frac{\lambda_{i}^{k_{i}}}{\left(k_{i}-1\right) !}\left(-\frac{\partial}{\partial s}\right)^{k_{i}-1}\left\{G_{N(T)}^{*}(s, z)\right\}\right|_{s=\lambda_{i}}
$$

where $G_{N(T)}^{*}(s, z)$ is given by (2.8). Thus we can obtain the expressions for the pmf and moments of $N(T)$ similar to (2.10) and (2.11). 
If we consider a mixture of exponential distributions for the CHT, or $k_{i}=1$ for all $i$ in (3.1), we have a hyperexponential $p d f$

$$
f_{T}(t)=\sum_{i=1}^{M} p_{i} \lambda_{i} e^{-\lambda_{i} t} ; \quad t \geq 0
$$

where $\sum_{i=1}^{M} p_{i}=1$. In this case, the coefficient of variation for $T$ is larger than unity, which is typical for data communication. Then the pgf of $N(T)$ is given by

$$
G_{N(T)}(z)=\left.\sum_{i=1}^{M} p_{i} \lambda_{i}\left\{G_{N(T)}^{*}(s, z)\right\}\right|_{s=\lambda_{i}}
$$

where $G_{N(T)}^{*}(s, z)$ is still given in $(2.8)$. Thus we get

$$
G_{N(T)}(z)=1+(z-1) \sum_{i=1}^{M} p_{i} \frac{f_{X_{1}}^{*}\left(\lambda_{i}\right)}{1-z f_{X_{2}}^{*}\left(\lambda_{i}\right)}
$$

where $T=\sum_{i=1}^{M} p_{i} T_{i}$ is the mixture of $M$ exponentially distributed random variables $T_{i}$ with mean $\mathbf{E}\left[T_{i}\right]=1 / \lambda_{i}$. The $j$ th derivative of this $p g f$ is given by

$$
\frac{d^{j}}{d z^{j}} G_{N(T)}(z)=j ! \sum_{i=1}^{M} p_{i} \frac{f_{X_{1}}^{*}\left(\lambda_{i}\right)\left[1-f_{X_{2}}^{*}\left(\lambda_{i}\right)\right]\left[f_{X_{2}}^{*}\left(\lambda_{i}\right)\right]^{j-1}}{\left[1-z f_{X_{2}}^{*}\left(\lambda_{i}\right)\right]^{j+1}} ; \quad j=1,2, \ldots
$$

Hence, the pmf of $N(T)$ is given by

$$
P[N(T)=j]=\left\{\begin{array}{ll}
1-\sum_{i=1}^{M} p_{i} f_{X_{1}}^{*}\left(\lambda_{i}\right) & ; j=0 \\
\sum_{i=1}^{M} p_{i} f_{X_{1}}^{*}\left(\lambda_{i}\right)\left[1-f_{X_{2}}^{*}\left(\lambda_{i}\right)\right]\left[f_{X_{2}}^{*}\left(\lambda_{i}\right)\right]^{j-1} & ; j=1,2, \ldots
\end{array} .\right.
$$

The $\ell$ th binomial moment of $N(T)$ is given by

$$
\mathbf{E}\left[\left(\begin{array}{c}
N(T) \\
\ell
\end{array}\right)\right]=\sum_{i=1}^{M} p_{i} \frac{f_{X_{1}}^{*}\left(\lambda_{i}\right)\left[f_{X_{2}}^{*}\left(\lambda_{i}\right)\right]^{\ell-1}}{\left[1-f_{X_{2}}^{*}\left(\lambda_{i}\right)\right]^{\ell}} \quad \ell=1,2, \ldots .
$$

Thus we have the mean

$$
\mathbf{E}[N(T)]=\sum_{i=1}^{M} p_{i} \frac{f_{X_{1}}^{*}\left(\lambda_{i}\right)}{1-f_{X_{2}}^{*}\left(\lambda_{i}\right)}
$$

The variance is given by

$$
\operatorname{Var}[N(T)]=2 \sum_{i=1}^{M} p_{i} \frac{f_{X_{1}}^{*}\left(\lambda_{i}\right) f_{X_{2}}^{*}\left(\lambda_{i}\right)}{\left[1-f_{X_{2}}^{*}\left(\lambda_{i}\right)\right]^{2}}+\mathbf{E}[N(T)]-\mathbf{E}^{2}[N(T)]
$$

We can obtain several interesting cases by specifying the Laplace transforms of the $p d f$ for both types of CRTs, as shown in the following subsections [14].

\subsection{Gamma distributed CRT}

We can apply the above formulas to any distribution for $X_{1}$ and $X_{2}$ with closed-form Laplace transforms. For instance, we can assume that CRTs $X_{1}$ and $X_{2}$ are gamma distributed with different scale parameters, namely, $X_{1}$ is gamma distributed with parameters $\left(\mu_{1}, \alpha_{1}\right)$, and 
$X_{2}$ has the parameters $\left(\mu_{2}, \alpha_{2}\right)$. Their mean values are given by $\mathbf{E}\left[X_{r}\right]=\alpha_{r} / \mu_{r}, r=1,2$, and their Laplace transforms are as follows:

$$
f_{X_{r}}^{*}(s)=\left(\frac{\mu_{r}}{s+\mu_{r}}\right)^{\alpha_{r}} ; \quad r=1,2 .
$$

Hence the $p m f$ for $N(T)$ is given by

$$
P[N(T)=j]=\left\{\begin{array}{cc}
1-\sum_{i=1}^{M} p_{i}\left(\frac{\rho_{1, i}}{\alpha_{1}^{-1}+\rho_{1, i}}\right)^{\alpha_{1}} ; & j=0 \\
\sum_{i=1}^{M} p_{i}\left(\frac{\rho_{1, i}}{\alpha_{1}^{-1}+\rho_{1, i}}\right)^{\alpha_{1}}\left[1-\left(\frac{\rho_{2, i}}{\alpha_{2}^{-1}+\rho_{2, i}}\right)^{\alpha_{2}}\right]\left(\frac{\rho_{2, i}}{\alpha_{2}^{-1}+\rho_{2, i}}\right)^{\alpha_{2}(j-1)} \\
j=1,2, \ldots
\end{array}\right.
$$

The $\ell$ th binomial moment of $N(T)$ is given by

$$
\mathbf{E}\left[\left(\begin{array}{c}
N(T) \\
\ell
\end{array}\right)\right]=\sum_{i=1}^{M} p_{i} \frac{\left(\frac{\rho_{1, i}}{\alpha_{1}^{-1}+\rho_{1, i}}\right)^{\alpha_{1}}}{\left(\frac{\rho_{2, i}}{\alpha_{2}^{-1}+\rho_{2, i}}\right)^{\alpha_{2}}\left[\left(\frac{\alpha_{2}^{-1}+\rho_{2, i}}{\rho_{2, i}}\right)^{\alpha_{2}}-1\right]^{\ell}} ; \quad \ell=1,2, \ldots .
$$

Therefore, the mean value is given by

$$
\mathbf{E}[N(T)]=\sum_{i=1}^{M} p_{i} \frac{\left(\frac{\rho_{1, i}}{\alpha_{1}^{-1}+\rho_{1, i}}\right)^{\alpha_{1}}}{1-\left(\frac{\rho_{2, i}}{\alpha_{2}^{-1}+\rho_{2, i}}\right)^{\alpha_{2}}}
$$

Let us assume that CRTs are exponentially distributed as in (2.20). This is just a particular case of the previous gamma distributed CRTs when $\alpha_{1}=\alpha_{2}=1$ in (3.11). The $p m f$ in (3.12) then reduces to

$$
P[N(T)=j]=\left\{\begin{array}{ll}
1-\sum_{i=1}^{M} p_{i} \frac{\rho_{1, i}}{1+\rho_{1, i}} & ; j=0 \\
\sum_{i=1}^{M} p_{i}\left(\frac{\rho_{1, i}}{1+\rho_{1, i}}\right)\left(\frac{1}{1+\rho_{2, i}}\right)\left(\frac{\rho_{2, i}}{1+\rho_{2, i}}\right)^{j-1} \quad ; j=1,2, \ldots
\end{array},\right.
$$

where $\rho_{r, i}=\mathbf{E}\left[T_{i}\right] / \mathbf{E}\left[X_{r}\right]=\mu_{r} / \lambda_{i}$ for $r=1,2$. The $\ell$ th binomial moment of $N(T)$ is given by

$$
\mathbf{E}\left[\left(\begin{array}{c}
N(T) \\
\ell
\end{array}\right)\right]=\sum_{i=1}^{M} p_{i} \rho_{1, i}\left(\frac{1+\rho_{2, i}}{1+\rho_{1, i}}\right) \rho_{2, i}^{\ell-1} ; \quad \ell=1,2, \ldots .
$$

Therefore, the mean value is given by

$$
\mathbf{E}[N(T)]=\sum_{i=1}^{M} p_{i} \rho_{1, i}\left(\frac{1+\rho_{2, i}}{1+\rho_{1, i}}\right)
$$

and the variance is given by

$$
\operatorname{Var}[N(T)]=2 \sum_{i=1}^{M} p_{i} \rho_{1, i} \rho_{2, i}\left(\frac{1+\rho_{2, i}}{1+\rho_{1, i}}\right)+\mathbf{E}[N(T)]-\mathbf{E}^{2}[N(T)] .
$$




\subsection{Generalized gamma distributed CRT}

According to Zonoozi and Dassanayake [19], the CRT for the first cell and that for the subsequent cells can be modeled by generalized gamma distributions with the $p d f$

$$
f_{X_{r}}(x)=\frac{c_{r} x^{\alpha_{r} c_{r}-1}}{b_{r}^{\alpha_{r} c_{r}} \Gamma\left(\alpha_{r}\right)} e^{-\left(x / b_{r}\right)^{c_{r}}} ; \quad x \geq 0, \alpha_{r}, b_{r}, c_{r}>0 ; \quad r=1,2
$$

where $\Gamma(\alpha)=\int_{0}^{\infty} u^{\alpha-1} e^{-u} d u, \alpha>0$, is the gamma function. In fact, by simulations of mobile patterns, a good matching has been produced by assuming that $\alpha_{1}=0.62, b_{1} \approx 1.85 R$, and $c_{1}=1.88$ for the first cell, and that $\alpha_{2}=2.31, b_{2} \approx 1.22 R$, and $c_{2}=1.72$ for the subsequent cells, where $R$ is the radius of circular cells. It is interesting to note that these values remain unchanged even for more general assumptions in the simulation such as the changes in velocity and direction of the user movement [19].

The $p d f$ in (3.19) may not have a rational Laplace transform, but its $k$ th moment about the origin is given by

$$
\mathbf{E}\left[X_{r}^{k}\right]=\frac{b_{r}{ }^{k} \Gamma\left(\alpha_{r}+k / c_{r}\right)}{\Gamma\left(\alpha_{r}\right)} ; \quad k=0,1,2, \ldots
$$

We can obtain several special cases of (3.19) by selecting the corresponding parameters. For instance, we can obtain the gamma distribution by choosing $c_{r}=1$ and $b_{r}=1 / \mu_{r}$, and from here the exponential distribution by letting $\alpha_{r}=1$.

We should remember that for a random variable $X$ with $p d f f_{X}(x)$, its Laplace transform $f_{X}^{*}(s)$ can be expanded in the moments of $X$ as follows:

$$
f_{X}^{*}(s)=\sum_{k=0}^{\infty}(-1)^{k} \frac{s^{k}}{k !} \mathbf{E}\left[X^{k}\right] .
$$

Hence, by using the moments in (3.20), we can expand $f_{X_{r}}^{*}\left(\lambda_{i}\right)$ as

$$
f_{X_{r}}^{*}\left(\lambda_{i}\right)=1+\sum_{k=1}^{\infty}(-1)^{k} \frac{G_{k, r}}{k ! \rho_{r, i}{ }^{k}} ; \quad r=1,2
$$

where

$$
G_{k, r}=\frac{\Gamma^{k-1}\left(\alpha_{r}\right) \Gamma\left(\alpha_{r}+k / c_{r}\right)}{\Gamma^{k}\left(\alpha_{r}+1 / c_{r}\right)} ; \quad r=1,2
$$

and

$$
\rho_{r, i}=\frac{\mathbf{E}\left[T_{i}\right]}{\mathbf{E}\left[X_{r}\right]}=\frac{\Gamma\left(\alpha_{r}\right)}{\lambda_{i} b_{r} \Gamma\left(\alpha_{r}+1 / c_{r}\right)} ; \quad r=1,2
$$

Note that the dependence on the parameters $\lambda_{i}$ and $b_{r}$ is concentrated only in the relative mobility ratio $\rho_{r, i}$ given in (3.24). The expansion in (3.22) can be substituted into (3.7) and (3.8) to obtain the pmf and the moments of $N(T)$, respectively. We also note that the series expansion for $f_{X}^{*}(s)$ in (3.21) is useful to obtain the $p m f$ and the moments of $N(T)$ for those distributions of $X$ that do not have closed-form expression for the Laplace transform.

\subsection{Circularly distributed CRT}

The hexagonal geometry for the wireless cells has been approximated by circles by Hong and Rappaport [5] and Yeung and Nanda [18]. They have derived the CRT distributions under the assumptions that the mobile users are uniformly distributed in the system and that they move in straight lines with direction uniformly distributed over $[0,2 \pi)$. The $p d f$ 
for the distance $Z_{1}$ from an arbitrary interior point of a circle with radius $R$ to its boundary in an arbitrary direction is given by [5, eq. (46)]

$$
f_{Z_{1}}(z)=\frac{2}{\pi R^{2}} \sqrt{R^{2}-\left(\frac{z}{2}\right)^{2}} ; \quad 0 \leq z \leq 2 R
$$

Hence the CRT in the first cell is given by the random variable $X_{1}:=Z_{1} / V$, where $V$ is the velocity of the user, which we will assume to be constant in the rest of this paper. In fact, this is a typical assumption also made in [5] and [18]. Under this assumption of constant velocity, the $k$ th moment of $X_{1}$ about the origin is given by

$$
\mathbf{E}\left[X_{1}{ }^{k}\right]=\mathbf{E}\left[\frac{Z_{1}{ }^{k}}{V^{k}}\right]=\frac{1}{V^{k}} \int_{z=0}^{2 R} z^{k} f_{Z_{1}}(z) d z
$$

or equivalently

$$
\mathbf{E}\left[X_{1}^{k}\right]=\frac{2}{\pi R^{2} V^{k}} \int_{z=0}^{2 R} z^{k} \sqrt{R^{2}-(z / 2)^{2}} d z=\frac{\Gamma\left(\frac{k+1}{2}\right)}{\sqrt{\pi} \Gamma\left(\frac{k}{2}+2\right)}\left(\frac{2 R}{V}\right)^{k} .
$$

Substituting (3.27) into (3.21), we obtain

$$
f_{X_{1}}^{*}\left(\lambda_{i}\right)=\sum_{k=0}^{\infty}(-1)^{k} \frac{M_{k, 1}}{k ! \rho_{1, i} k}
$$

where

$$
M_{k, 1}=\pi^{k-\frac{1}{2}}\left(\frac{3}{4}\right)^{k} \frac{\Gamma\left(\frac{k+1}{2}\right)}{\Gamma\left(\frac{k}{2}+2\right)} \quad ; \quad \rho_{1, i}=\frac{\mathbf{E}\left[T_{i}\right]}{\mathbf{E}\left[X_{1}\right]}=\frac{3 \pi V}{8 R \lambda_{i}}
$$

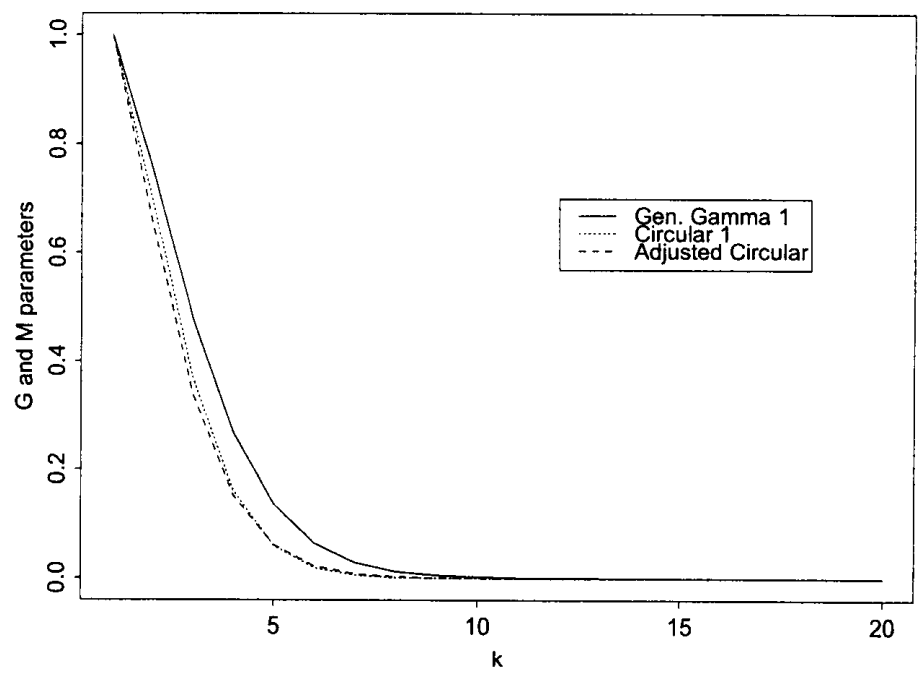

Figure 2: Plots of $G_{k, 1} / k$ ! and $M_{k, 1} / k$ ! as a function of $k$ 


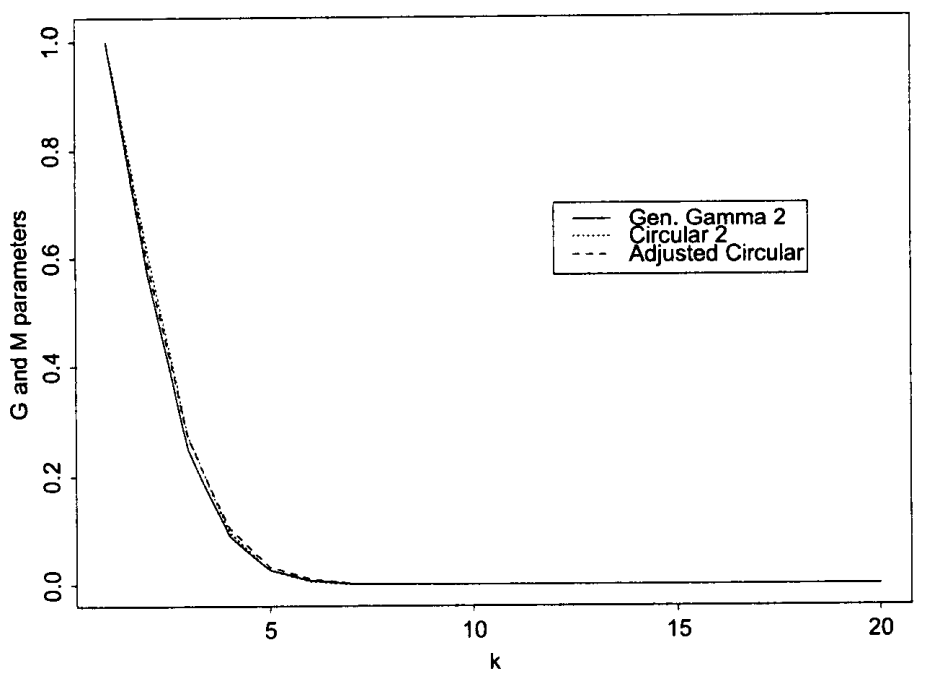

Figure 3: Plots of $G_{k, 2} / k$ ! and $M_{k, 2} / k$ ! as a function of $k$

Similarly, the $p d f$ for the distance $Z_{2}$ from an arbitrary point on the boundary of a circle with radius $R$ at which the mobile user enters the cell to another point on the boundary in a random direction at which he exits from the cell in a straight line is given by [5, eq.(51)]

$$
f_{Z_{2}}(z)=\frac{2}{\pi \sqrt{4 R^{2}-z^{2}}} ; \quad 0 \leq z<2 R
$$

We note that the path of a user in this case is equivalent to the random chord of a circle which has been studied in the field of geometrical probability [10, p.198]. There are several ways to define the randomness of the chord which lead to different probability measures. The most appropriate one in the modeling of user movement in cellular systems seems to be that the direction of the path is uniformly distributed over $[0,2 \pi)$, which is called $S$ randomness [1]. In fact, the $p d f$ in (3.30) is exactly the same as the $p d f$ for the length of a random chord of a circle with radius $R$ in the sense of $S$-randomness [10, eq.(2.3.41), p.198]. Then the $k$ th moment of the CRT $X_{2}:=Z_{2} / V$ for the second and subsequent cells is given by

$$
\mathbf{E}\left[X_{2}^{k}\right]=\frac{\Gamma\left(\frac{k+1}{2}\right)}{\sqrt{\pi} \Gamma\left(\frac{k+2}{2}\right)}\left(\frac{2 R}{V}\right)^{k}
$$

Substituting (3.31) into (3.21), we obtain

$$
f_{X_{2}}^{*}\left(\lambda_{i}\right)=\sum_{k=0}^{\infty}(-1)^{k} \frac{M_{k, 2}}{k ! \rho_{2, i}{ }^{k}}
$$

where

$$
M_{k, 2}=\frac{\pi^{k-\frac{1}{2}}}{2^{k}} \frac{\Gamma\left(\frac{k+1}{2}\right)}{\Gamma\left(\frac{k}{2}+1\right)} \quad ; \quad \rho_{2, i}=\frac{\mathbf{E}\left[T_{i}\right]}{\mathbf{E}\left[X_{2}\right]}=\frac{\pi V}{4 R \lambda_{i}} .
$$

Let us call the above distributions for $X_{1}$ and $X_{2}$ the circular distributions.

We note that the dependence of $f_{X_{r}}^{*}\left(\lambda_{i}\right)$ on the parameters $\lambda_{i}, V$, and $R$ is all concentrated in the relative mobility ratios $\rho_{r, i}$ given in (3.29) and (3.33). We also observe that 
the series expansions in the above for the circular distributions are similar to the series expansion of the generalized gamma distribution in (3.22). In the above derivation, the parameter $M_{k, r}$ is a function of only $k$ for $r=1,2$. Thus we can match the series expansion for the generalized gamma distribution in (3.22) with those in (3.28) and (3.32) for the circular distributions. We have found that the parameters reported in [19] for $\alpha_{r}$ and $c_{r}$ produce reasonable matching, as shown in Figures 2 and 3 . We can present better matching to the circularly distributed case by adjusting the parameters of the generalized gamma distribution. While many combinations of the values for $\alpha_{r}$ and $c_{r}$ are possible, we show in Figure 2 only one of them by fixing $c_{1}=1.88$ and finding $\alpha_{1}=1.0$. Similarly, in Figure 3 we fix $c_{2}=1.72$ and find $\alpha_{2}=1.85$ for the best matching. We may call the generalized gamma distribution with these parameter values the adjusted circular distribution. In Figure 2 the generalized gamma case assumes $\alpha_{1}=0.62$ and $c_{1}=1.88$, whereas $\alpha_{2}=2.31$ and $c_{2}=1.72$ in Figure 3.

It may also be interesting to compare the $p d f f_{Z_{1}}(z)$ for $Z_{1}$ given in (3.25) and the $p d f$ for $\hat{Z}_{2}$, the residual life of $Z_{2}$, which is given by

$$
\begin{aligned}
f_{\hat{Z}_{2}}(z) & =\frac{1}{\mathbf{E}\left[Z_{2}\right]}\left[1-\int_{0}^{z} f_{Z_{2}}(x) d x\right]=\frac{\pi}{4 R}\left[1-\frac{2}{\pi} \arcsin \left(\frac{z}{2 R}\right)\right] \\
& =\frac{1}{2 R} \arccos \left(\frac{z}{2 R}\right) ; \quad 0 \leq z \leq 2 R .
\end{aligned}
$$

As shown in Figure 4, they are different. This fact justifies the analysis of considering a distribution for the first CRT different from the distribution of the subsequent CRTs in this paper.

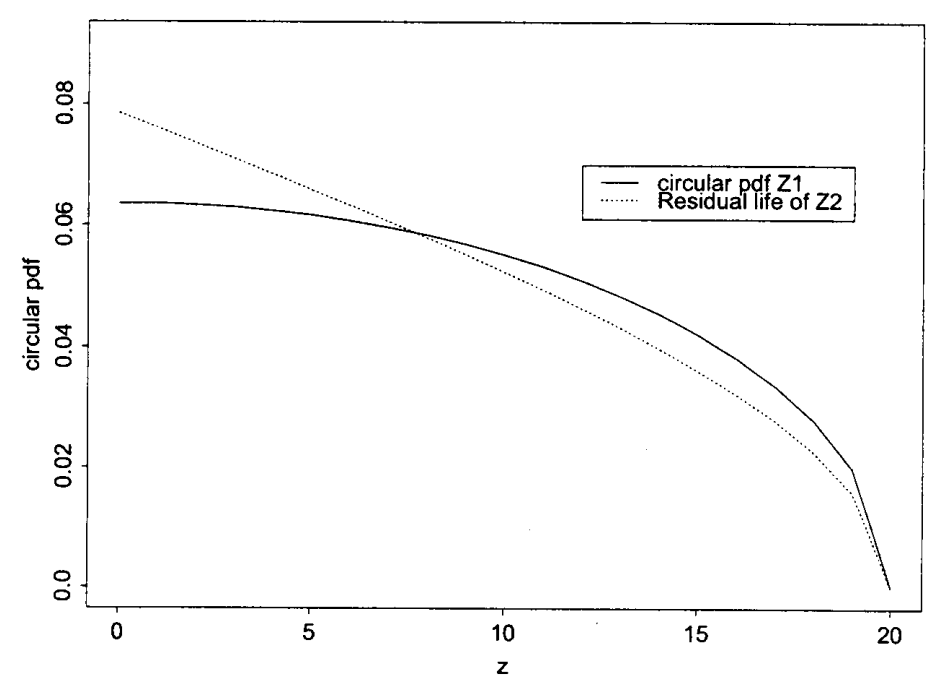

Figure 4: Plots of the $p d f f_{Z_{1}}(z)$ for $Z_{1}$ and the $p d f$ for the residual life of $Z_{2}$

\section{Generalized Delayed Renewal Process for CRTs}

Let us generalize the delayed renewal process of CRTs so that each CRT $X_{1}, X_{2}, \ldots$ may have different distribution. 
Suppose that the first $R$ CRTs $X_{1}, X_{2}, \ldots, X_{R}$ may have different distributions for which the Laplace transforms of the $p d f$ are given by $f_{X_{1}}^{*}(s), f_{X_{2}}^{*}(s), \ldots, f_{X_{R}}^{*}(s)$, respectively, and that the subsequent CRTs $X_{R+1}, X_{R+2}, \ldots$ have the same distribution as $X_{R}$. As special cases of this process, we have an ordinary renewal process for $R=1$, a delayed renewal process for $R=2$, and the case in which all CRTs are distinct for $R=\infty$.

For this process, from $[2$, p.37,eq.(3)], we have

$$
\begin{aligned}
G_{N(T)}^{*}(s, z) & =\frac{1}{s}+\frac{z-1}{s}\left[\sum_{j=1}^{R-1} z^{j-1} \prod_{r=1}^{j} f_{X_{r}}^{*}(s)+\sum_{j=R}^{\infty} z^{j-1} F_{R}^{*}(s)\left[f_{X_{R}}^{*}(s)\right]^{j-R}\right] \\
& =\frac{1}{s}+\frac{z-1}{s} \sum_{j=1}^{R-1} z^{j-1} \prod_{r=1}^{j} f_{X_{r}}^{*}(s)+\frac{(z-1) z^{R-1} F_{R}^{*}(s)}{s\left[1-z f_{X_{R}}^{*}(s)\right]}
\end{aligned}
$$

where we have introduced for notational convenience

$$
F_{R}^{*}(s):=\prod_{r=1}^{R} f_{X_{r}}^{*}(s)
$$

This result is a generalization of (2.8).

\subsection{Exponentially distributed CHT}

If the CHT $T$ is exponentially distributed with mean $\mathbf{E}[T]=1 / \lambda$ as in (2.13), we have the $p g f$ for $N(T)$ as

$$
\begin{aligned}
G_{N(T)}(z) & =\left.\lambda G_{N(T)}^{*}(s, z)\right|_{s=\lambda} \\
& =1+(z-1) \sum_{j=1}^{R-1} z^{j-1} \prod_{r=1}^{j} f_{X_{\tau}}^{*}(\lambda)+\frac{(z-1) z^{R-1} F_{R}^{*}(\lambda)}{1-z f_{X_{R}}^{*}(\lambda)} .
\end{aligned}
$$

It is straightforward as before to obtain the pmf and moments of $N(T)$ from (4.3). As the coefficient of $z^{j}$ in the expansion of (4.3) in powers of $z$, the pmf of $N(T)$ is given by

$$
P[N(T)=j]= \begin{cases}1-f_{X_{1}}^{*}(\lambda) & ; j=0 \\ {\left[1-f_{X_{j+1}}^{*}(\lambda)\right] \prod_{r=1}^{j} f_{X_{r}}^{*}(\lambda)} & ; 1 \leq j \leq R-1 \\ F_{R}^{*}(\lambda)\left[1-f_{X_{R}}^{*}(\lambda)\right]\left[f_{X_{R}}^{*}(\lambda)\right]^{j-R} & ; j \geq R\end{cases}
$$

As the coefficient of $(z-1)^{\ell}$ in the expansion of (4.3) in powers of $z-1$, the $\ell$ th binomial moment of $N(T)$ is given by

$$
\mathbf{E}\left[\left(\begin{array}{c}
N(T) \\
\ell
\end{array}\right)\right]=\left\{\begin{array}{cc}
\sum_{j=\ell}^{R-1}\left(\begin{array}{c}
j-1 \\
\ell-1
\end{array}\right) \prod_{r=1}^{j} f_{X_{r}}^{*}(\lambda)+F_{R}^{*}(\lambda) \sum_{j=0}^{\ell-1}\left(\begin{array}{c}
R-1 \\
\ell-j-1
\end{array}\right) \frac{\left[f_{X_{R}}^{*}(\lambda)\right]^{j}}{\left[1-f_{X_{R}}^{*}(\lambda)\right]^{j+1}} & 1 \leq \ell \leq R-1 \\
F_{R}^{*}(\lambda) \sum_{j=\ell-R}^{\ell-1}\left(\begin{array}{c}
R-1 \\
\ell-j-1
\end{array}\right) \frac{\left[f_{X_{R}}^{*}(\lambda)\right]^{j}}{\left[1-f_{X_{R}}^{*}(\lambda)\right]^{j+1}} ; \quad \ell \geq R
\end{array}\right.
$$


In particular, the mean is given by

$$
\mathbf{E}[N(T)]=\sum_{j=1}^{R-1} \prod_{r=1}^{j} f_{X_{r}}^{*}(\lambda)+\frac{F_{R}^{*}(\lambda)}{1-f_{X_{R}}^{*}(\lambda)} .
$$

All the above expressions reduce to those in Section 2 when $R=2$.

For $R=\infty$, by assuming that $\lim _{R \rightarrow \infty} F_{R}^{*}(\lambda)=0$ for $\lambda>0$, the pgf of $N(T)$ is given by

$$
G_{N(T)}(z)=1+(z-1) \sum_{j=1}^{\infty} z^{j-1} \prod_{r=1}^{j} f_{X_{r}}^{*}(\lambda)
$$

Thus we have

$$
P[N(T)=j]= \begin{cases}1-f_{X_{1}}^{*}(\lambda) & ; j=0 \\ {\left[1-f_{X_{j+1}}^{*}(\lambda)\right] \prod_{r=1}^{j} f_{X_{r}}^{*}(\lambda) ; j=1,2, \ldots}\end{cases}
$$

and

$$
\mathbf{E}\left[\left(\begin{array}{c}
N(T) \\
\ell
\end{array}\right)\right]=\sum_{j=\ell}^{\infty}\left(\begin{array}{c}
j-1 \\
\ell-1
\end{array}\right) \prod_{r=1}^{j} f_{X_{r}}^{*}(\lambda) ; \quad \ell=1,2, \ldots .
$$

\subsection{General CHT and exponentially distributed CRTs}

Now, we consider a general $p d f$ for the CHT and exponentially distributed CRTs as

$$
f_{X_{r}}^{*}(s)=\frac{\mu_{r}}{s+\mu_{r}} ; \quad r=1,2, \ldots, R .
$$

Let us assume for simplicity that all $\mu_{r}$ 's are distinct. Substituting (4.10) into (4.1) and expanding in partial fractions in $s$ yields

$$
G_{N(T)}^{*}(s, z)=(1-z) \sum_{r=1}^{R-1} \frac{B_{r}(z)}{s+\mu_{r}}+\frac{C(z)}{s+\mu_{R}(1-z)},
$$

where

$$
B_{r}(z):=\frac{\left(\mu_{r}-\mu_{R}+\mu_{R} z\right) \sum_{j=r}^{R-2} z^{j-1}\left[\prod_{i=1}^{j} \mu_{i}\right]\left[\prod_{i=j+1}^{R-1}\left(\mu_{i}-\mu_{r}\right)\right]+z^{R-2}\left(\mu_{r}-\mu_{R}\right) \prod_{j=1}^{R-1} \mu_{j}}{\mu_{r}\left(\mu_{r}-\mu_{R}+\mu_{R} z\right) \prod_{j=1(j \neq r)}^{R-1}\left(\mu_{j}-\mu_{r}\right)}
$$

and

$$
C(z):=z^{R-1} \prod_{j=1}^{R-1} \frac{\mu_{j}}{\mu_{j}-\mu_{R}+\mu_{R} z} .
$$

Substituting the inversion of (4.11) into (2.1), we obtain the $p g f$ of $N(T)$ as

$$
G_{N(T)}(z)=(1-z) \sum_{r=1}^{R-1} B_{r}(z) f_{T}^{*}\left(\mu_{r}\right)+C(z) f_{T}^{*}\left[\mu_{R}(1-z)\right]
$$


where $f_{T}^{*}(s)$ is the Laplace transform of the $p d f$ for the generally distributed CHT $T$.

It is possible to derive the pmf and moments of $N(T)$ from (4.14). For example, the mean is given by

$$
\mathbf{E}[N(T)]=-\sum_{r=1}^{R-1} B_{r}(1) f_{T}^{*}\left(\mu_{r}\right)+R+\mu_{R}\left(\mathbf{E}[T]-\sum_{r=1}^{R} \frac{1}{\mu_{r}}\right)
$$

where

$$
B_{r}(1)=\frac{\mu_{r} \sum_{j=r}^{R-2}\left[\prod_{i=1}^{j} \mu_{i}\right]\left[\prod_{i=j+1}^{R-1}\left(\mu_{i}-\mu_{r}\right)\right]+\left(\mu_{r}-\mu_{R}\right) \prod_{j=1}^{R-1} \mu_{j}}{\mu_{r}^{2} \prod_{j=1(j \neq r)}^{R-1}\left(\mu_{j}-\mu_{r}\right)} ; \quad r=1,2, \ldots, R-1 .
$$

\subsection{General CHT and exponentially distributed delayed renewal process for CRTs}

In this section, we consider a general $p d f$ for the CHT and the delayed renewal process for the CRTs that are exponentially distributed as in (2.20). This is a special case of the Section 4.2 for $R=2$.

The $p g f$ for $N(T)$ is given by

$$
G_{N(T)}(z)=\frac{\left(\mu_{2}-\mu_{1}\right)(z-1)}{\mu_{1}+\mu_{2}(z-1)} f_{T}^{*}\left(\mu_{1}\right)+\frac{\mu_{1} z}{\mu_{1}+\mu_{2}(z-1)} f_{T}^{*}\left[\mu_{2}(1-z)\right]
$$

which follows by substituting $R=2$ into (4.14).

We need the $j$ th derivative of $G_{N(T)}(z)$ in order to find the $p m f$ and the moments of $N(T)$. It is given by

$$
\begin{aligned}
\frac{d^{j} G_{N(T)}(z)}{d z^{j}} & =\frac{(-1)^{j-1} j ! \mu_{1}\left(\mu_{2}-\mu_{1}\right) \mu_{2}^{j-1}}{\left[\mu_{1}+\mu_{2}(z-1)\right]^{j+1}} f_{T}^{*}\left(\mu_{1}\right) \\
& +(-1)^{j} j ! \mu_{1}\left(\mu_{2}-\mu_{1}\right) \mu_{2}^{j-1} \sum_{i=0}^{j-1} \frac{f_{T}^{*(i)}\left[\mu_{2}(1-z)\right]}{i !\left[\mu_{1}+\mu_{2}(z-1)\right]^{j-i+1}} \\
& +\frac{(-1)^{j} \mu_{1} \mu_{2}^{j} z}{\mu_{1}+\mu_{2}(z-1)} f_{T}^{*(j)}\left[\mu_{2}(1-z)\right] ; \quad j=1,2, \ldots,
\end{aligned}
$$

where

$$
f_{T}^{*(j)}(s):=\frac{d^{j} f_{T}^{*}(s)}{d s^{j}}=(-1)^{j} \int_{t=0}^{\infty} t^{j} e^{-s t} f_{T}(t) d t ; \quad j=1,2, \ldots
$$

From (4.18), we find the pmf for $N(T)$ as

$$
P[N(T)=j]=\left\{\begin{array}{ll}
f_{T}^{*}\left(\mu_{1}\right) & ; j=0 \\
\frac{\mu_{1} \mu_{2}^{j-1}}{\left(\mu_{2}-\mu_{1}\right)^{j}}\left[f_{T}^{*}\left(\mu_{1}\right)-\sum_{i=0}^{j-1} \frac{\left(\mu_{1}-\mu_{2}\right)^{i}}{i !} f_{T}^{*(j)}\left(\mu_{2}\right)\right] & ; j=1,2, \ldots .
\end{array} .\right.
$$

The th binomial moment of $N(T)$ is given by

$$
\begin{aligned}
\mathbf{E}\left[\left(\begin{array}{c}
N(T) \\
\ell
\end{array}\right)\right] & =\left(1-\frac{\mu_{1}}{\mu_{2}}\right)\left(\frac{\mu_{2}}{\mu_{1}}\right)^{\ell}\left\{(-1)^{\ell-1} f_{T}^{*}\left(\mu_{1}\right)+\sum_{i=0}^{\ell-1} \frac{(-1)^{\ell-i}}{i !} \mu_{1}^{i} \mathbf{E}\left[T^{i}\right]\right\} \\
& +\frac{\mu_{2}^{\ell}}{\ell !} \mathbf{E}\left[T^{\ell}\right] ; \quad \ell=1,2, \ldots
\end{aligned}
$$


For example, the mean is given by

$$
\mathbf{E}[N(T)]=\frac{\mu_{2}-\mu_{1}}{\mu_{1}}\left[f_{T}^{*}\left(\mu_{1}\right)-1\right]+\mu_{2} \mathbf{E}[T] .
$$

If the CHT is exponentially distributed as in (2.13), we recover the results in Section 2.1 .

\section{Numerical Results}

In previous sections we have obtained many closed-form solutions for the $p m f$ and statistical moments of the handover distribution for a variety of CHT and CRT combinations. Now, we will present some numerical examples of $\mathbf{E}[N(T)]$ to gain some insights into the mean value of handovers as a function of the mobility ratio $\rho$. There are many parameters involved in some of the models, so we only analyze a representative sample of all of them.

In Figure 5 we plot $\mathbf{E}[N(T)]$ for the EE model, i.e., exponential CHT and exponential CRTs, where $\rho_{2}=3 \rho_{1}$. We have also plotted four types of the HE model (hyperexponential CHT and exponential CRT). In all the cases, we only consider a mixture of two exponentials, i.e., $M=2$ in (3.1). For the HE1 model, we have set $p_{1}=0.1, \rho_{11}=\rho_{1}, \rho_{12}=3 \rho_{1}, \rho_{21}=\frac{2}{3} \rho_{1}$, and $\rho_{22}=2 \rho_{1}$. In the HE2 model we have changed $p_{1}$ to 0.5 , and in the HE3 model it has been set to 0.9 . The HE4 model has the same parameters as HE2 except that $\rho_{12}=\frac{3}{10} \rho_{1}$ and $\rho_{22}=\frac{2}{10} \rho_{1}$. It is easily seen from (2.25) that $\mathbf{E}[N(T)] \simeq K \rho_{1}$ when $\rho_{2}=K \rho_{1}$ and $\rho_{1} \gg 1$ for the EE model. A similar analysis of (3.17) shows that $\mathbf{E}[N(T)] \simeq \rho_{1}\left(2-\frac{4}{3} p_{1}\right)$ for the HE1, HE2, and HE3 models and that $\mathbf{E}[N(T)] \simeq \rho_{1}\left(\frac{1}{5}+\frac{7}{15} p_{1}\right)$ for the HE4 model, meaning the linear growth of $\mathbf{E}[N(T)]$ as $\rho_{1}$ increases.

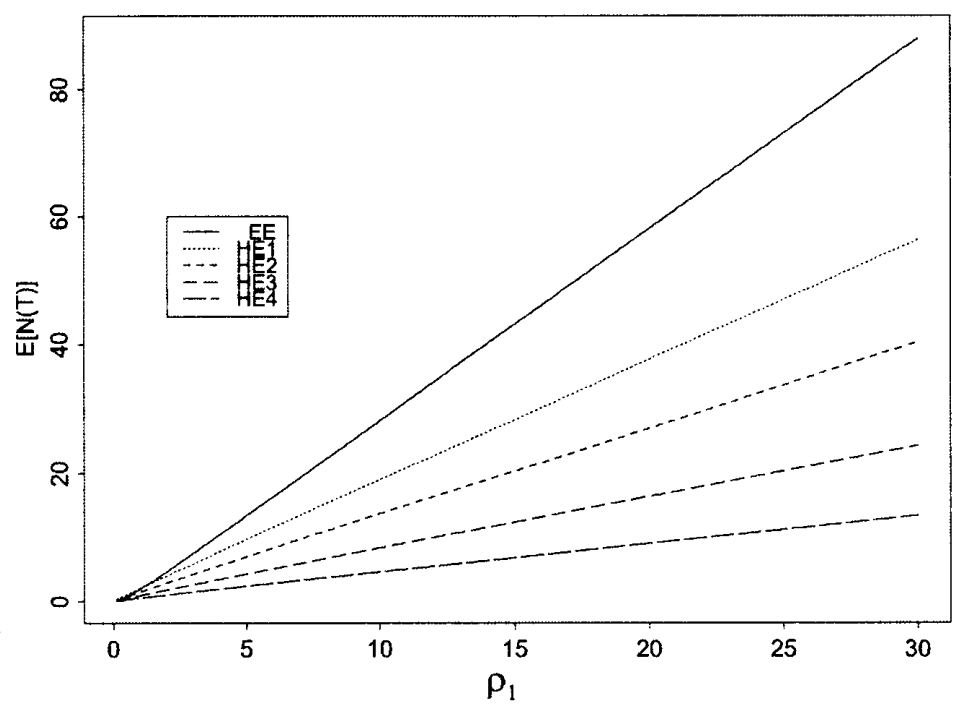

Figure 5: Plots of $\mathbf{E}[N(T)]$ as a function of $\rho_{1}$ for the $\mathrm{EE}$ and HE models

We also plot the EE model in Figure 6 in order to make some comparisons with the HC model, i.e., hyperexponential CHT and circular CRTs. We consider the cases of $p_{1}=0.1,0.5$ and 0.9 . In addition, we set the mobility rates to $\rho_{11}=\rho_{1}, \rho_{21}=\frac{2}{3} \rho_{1}, \rho_{12}=3 \rho_{1}$, and $\rho_{22}=2 \rho_{1}$. It is interesting to notice that $\mathbf{E}[N(T)]$ is a linear function of $\rho_{1}$ and that the slope decreases as $p_{1}$ increases. We have not shown $\mathbf{E}[N(T)]$ for the hyperexponential $\mathrm{CHT}$ and generalized gamma CRTs model with the parameter values adjusted for the circular CRTs as discussed in Section 3.3. However, there is a very good matching with the HC 
model for all cases of $p_{1}$ (the plots overlap) as a result of the good matching shown in Figures 2 and 3.

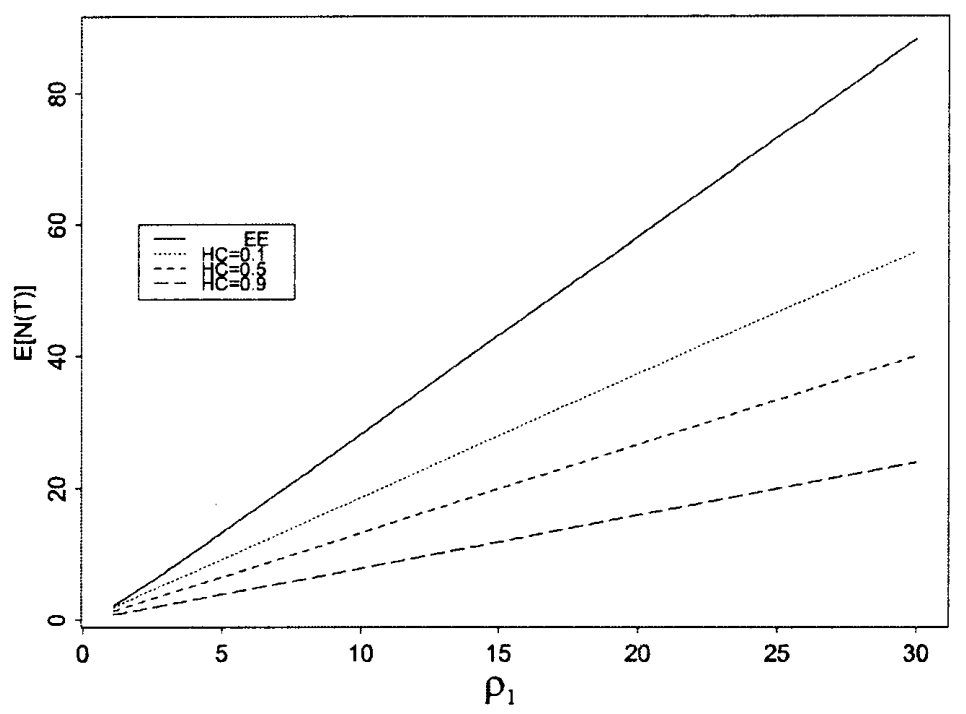

Figure 6: Plots of $\mathrm{E}[N(T)]$ as a function of $\rho_{1}$ for the $\mathrm{EE}$ and $\mathrm{HC}$ models

Finally, we define the GE model by assuming the gamma distributed CHT and the exponential CRTs. The Laplace transform of the gamma $p d f$ for the CHT is given by

$$
f_{T}^{*}(s)=\left(\frac{\lambda}{s+\lambda}\right)^{\alpha} .
$$

Thus, by substituting (5.1) into (4.22) we obtain

$$
\mathbf{E}[N(T)]=\left(\frac{\rho_{2}}{\rho_{1}}-1\right)\left[\left(\frac{\alpha}{\rho_{1}+\alpha}\right)^{\alpha}-1\right]+\rho_{2}
$$

where $\rho_{i}=\alpha \mu_{i} / \lambda, \quad(i=1,2)$. In Figure 7 , we plot $\mathbf{E}[N(T)]$ in (5.2) for $\rho_{2}=0.1 \rho_{1}$ and $\alpha=1$ (EE model), 1.5, 2.5, and 5.5. For all these cases $\mathbf{E}[N(T)]$ grows as $\rho_{1}$ increases, but the plots almost overlap, meaning that the influence of $\alpha$ in the gamma distribution for the CHT is negligible. This is because $\mathbf{E}[N(T)] \simeq 1-\left(\rho_{2} / \rho_{1}\right)+\rho_{2}$ when $\rho_{1} \gg 1$ in (5.2).

\section{Conclusions}

In this paper, we have derived explicit forms for the $p m f$ and the statistical moments of the number of handovers during a random call holding time (CHT) when the CHT distribution is well-fitted by a mixture of exponential distributions while the cell residence time (CRT) is arbitrarily distributed. As specific distributions for the CRT, we have dealt with exponential, gamma, and generalized gamma distributions as well as the distribution that comes from the length of a random chord of a circle for a model of circular cells. We have provided good numerical matching between the generalized gamma distribution and the circular distribution, which improves the result in [19]. We have also considered the case of a general CHT and exponentially distributed CRTs.

In the present study, we have assumed that the first wireless cell has a different CRT distribution from those for the subsequent cells. This model may be interesting for situations where the call originates in a picocell and the portable moves into the microcellular 


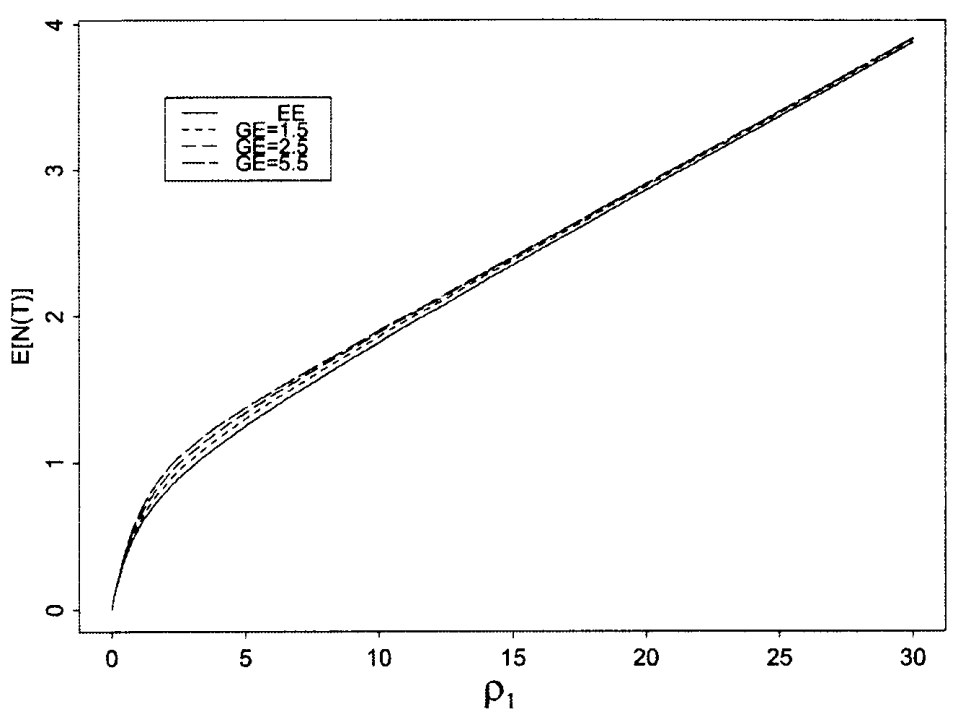

Figure 7: Plots of $\mathbf{E}[N(T)]$ as a function of $\rho_{1}$ for the EE and GE models

environment or vice versa. Our mathematical framework is the delayed renewal process [14] as a generalization of the equilibrium renewal process studied in $[13,15,17]$ in the homogeneous environment. This means that all but the first CRTs have the same probability distribution while the first CRT corresponds to its residual life. We have also presented the case of an irregular topology, which means that the CRT distributions may be different for all cells.

Many other interesting combinations of the CHT and CRT distributions can be handled in the same framework. We also remark that obtaining the $p m f$ and the moments for the number of handovers during a call session is an important step for obtaining other performance measures, including the probability of completing a call and the handover traffic rate, for mobile communication networks $[9,14,15]$. In addition, this analysis is important to obtain performance measures in dynamic mobility management, e.g., movement-based schemes $[6,7,16]$.

Acknowledgements: The first author thanks the partial support provided by the Japan Society for the Promotion of Science (JSPS) and Conacyt for his visit to the University of Tsukuba. He also thanks ITESM, Campus Monterrey, through the Research Chair in Telecommunications, for the support provided in the development of this work.

\section{References}

[1] R. Coleman: Random paths through convex bodies. Journal of Applied Probability, 6 (1969), 430-441.

[2] D.R. Cox: Renewal Theory (Methuen, London, 1962).

[3] Y. Fang, I. Chlamtac and Y.-B. Lin: Call performance for a PCS network. IEEE Journal on Selected Areas in Communications, 15 (1997), 1568-1581.

[4] Y. Fang, I. Chlamtac and Y.-B. Lin: Channel occupancy times and handoff rate for mobile computing and PCS networks. IEEE Transactions on Computers, 47 (1998), 679-692. 
[5] D. Hong and S.S. Rappaport: Traffic model and performance analysis for cellular mobile radio telephone systems with prioritized and nonprioritized handoff procedures. IEEE Transactions on Vehicular Technology, VT-35 (1986), 77-92.

[6] J. Li, H. Kameda and K. Li: Optimal Dynamic Mobility Management for PCS Networks. IEEE/ACM Transactions on Networking, 8, No.3, (2000), 319-327.

[7] J. Li, Y. Pan and X. Jia: Analysis of Dynamic Location Management for PCS Networks. IEEE Transactions on Vehicular Technology, 51, No.5, (2002), 1109-1119.

[8] Y.-B. Lin: Impact of PCS handoff response time. IEEE Communications Letters, 1 (1997), 160-162.

[9] Y.-B. Lin, S. Mohan and A. Noerpel: Queueing priority channel assignment strategies for handoff and initial access for a PCS network. IEEE Transactions on Vehicular Technology, 43 (1994), 704-712.

[10] A.M. Mathai: An Introduction to Geometrical Probability: Distributional Aspects with Applications (Gordon and Breach Science Publishers, 1999).

[11] S. Nanda: Teletraffic models for urban and suburban microcells: Cell sizes and handhoff rates. IEEE Transactions on Vehicular Technology, 42 (1993), 673-682.

[12] P.V. Orlik and S.S. Rappaport: Traffic performance and mobility modeling of cellular communications with mixed platforms and highly variable mobilities. Proceedings of the IEEE, 86 (1998), 1464-1479.

[13] R.M. Rodríguez-Dagnino: Handoff analysis in wireless multimedia networks. SPIE Conference on Performance and Control of Network Systems II, 3530 (1998), 76-84.

[14] R.M. Rodríguez-Dagnino, G. Hernández-Lozano and H. Takagi: Wireless handover distributions in mixed platforms with multimedia services. SPIE Conference on Internet Quality and Performance and Control of Network Systems, 4211 (2000), 59-69.

[15] R.M. Rodríguez-Dagnino and C.A. Leyva-Valenzuela: Performance analysis in broadband wireless networks. SPIE Conference on Performance and Control of Network Systems III, 3841 (1999), 220-228.

[16] R.M. Rodríguez-Dagnino, J.J. Ruiz-Cedillo and H. Takagi: Dynamic mobility management for cellular networks: A delayed renewal process approach. IEICE Transactions on Communications, E85-B, No.6, (2002), 1069-1074.

[17] R.M. Rodríguez-Dagnino and H. Takagi: Counting handovers in a cellular mobile communication network: equilibrium renewal process approach. Performance Evaluation, 52, Issues 2-3, (2003), 153-174.

[18] K.L. Yeung and S. Nanda: Channel management in microcell/macrocell cellular radio systems. IEEE Transactions on Vehicular Technology, 45 (1996), 601-612.

[19] M.M. Zonoozi and P. Dassanayake: User mobility modeling and characterization of mobility patterns. IEEE Journal on Selected Areas in Communications, 15 (1997), 1239-1252.

Ramón Martín Rodríguez-Dagnino

Monterrey Institute of Technology (ITESM) Centro de Electrónica y Telecomunicaciones Sucursal de correos "J". C.P. 64849

Monterrey, N.L., México.

E-mail: rmrodrig@itesm.mx 